\title{
Landing in Ski Jumping: A Review About its Biomechanics and the Connected Injuries
}

\author{
Veronica Bessone $^{1}$ D $\cdot$ Ansgar Schwirtz $^{1}$
}

Received: 12 May 2020 / Accepted: 30 October 2020 / Published online: 14 July 2021

(c) The Author(s) 2021

\begin{abstract}
The present review deals with the current scientific knowledge related with ski jump landing. A specific focus is given on the landing biomechanics, the methods utilized for its analysis and the injuries connected to the landing phase. Despite the demonstrated importance for the safety and the performance of ski jumpers, the landing and its preparation are rarely investigated. In this paper, after having firstly described the execution of landing and its preparation and the reason why is important to analyze it, an overview of the current status of the research related to the landing biomechanics is reported (kinetics, kinematics, electromyographic activation, aerodynamics, computer simulation). The third part describes the methods and technologies utilized in literature to analyze the landing and its preparation (video cameras, inertial sensors, force insoles, wind tunnel and computer simulation). After that, an overview of the injuries related to landing is reported. The final section proposes future research in the field of biomechanics of ski jump landing in different fields, such as computer simulations, kinematic analysis, equipment development and biomechanics of female athletes.
\end{abstract}

Keywords Feedback $\cdot$ Sensors $\cdot$ Prevention $\cdot$ Kinetics $\cdot$ Kinematics $\cdot$ Winter sports

\section{Introduction}

Ski jumping is a winter sport in which the execution of one of its different phases (in-run, take-off, early flight, flight, landing preparation and landing impact) is influencing the subsequent one and, consequently, the overall performance. The biomechanics of the last two phases (landing impact and its preparation) plays a central role for improving the performance and for reducing the injuries $[17,23,38,41$, 44, 47, 52, 53] (Fig. 1). However, despite the importance for the performance and the safety of ski jumpers, the number of publications related to landing is limited, mainly due to technological problems (such as the presence of wires and the weight of the equipment) [43].

As stated in the FIS International Competition Rules (ICR) of Ski Jumping [12], for executing the landing, the athlete should act as follows. "From a stable flight position, raises head and upper body, moves the arms on the sides

Veronica Bessone

veronica.bessone@tum.de

1 Department of Biomechanics in Sports, Faculty of Sport and Health Sciences, Technical University of Munich, Munich, Bavaria, Germany
[...] and turns the skis into a parallel position", then just before the touch-down "splitting the legs and bending the knees". Reduce the impact by means of muscle power and "increase the distance between the legs and bend the back leg [...], (telemark position) with the skis parallel and obtain the pressure equal on both legs and [...] stretch both arms horizontally and forwards upwards" (Fig. 2). The athlete should perform the connection between the phases with a smooth movement for optimizing the performance. In fact, the technical execution of the telemark landing is evaluated by five judges with additional points, influencing the final score of the performance (FIS ICR Ski Jumping) [12]. Besides the telemark landing, the athlete can also land using a parallel leg landing, i.e. landing with the two feet at the same height in a squat position. Differently from the telemark landing, the parallel leg landing negatively influences the technical score given by the judges. Further technical adaptations about the landing are left to the athletes' experience and coaches' suggestions due to the lack of studies. However, knowing the most important biomechanical predictors that lead to injuries and improve the landing execution could permit to focus training and technical suggestions.

The importance of landing (both the impact and the landing preparation phases) seems underestimated by athletes 


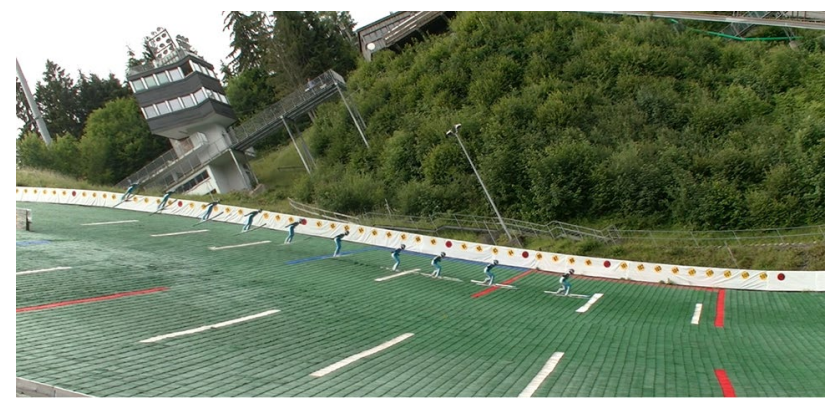

Fig. 1 Progression of a landing preparation phase obtained from a video (normal ski jumping hill in Oberstdorf, Germany)

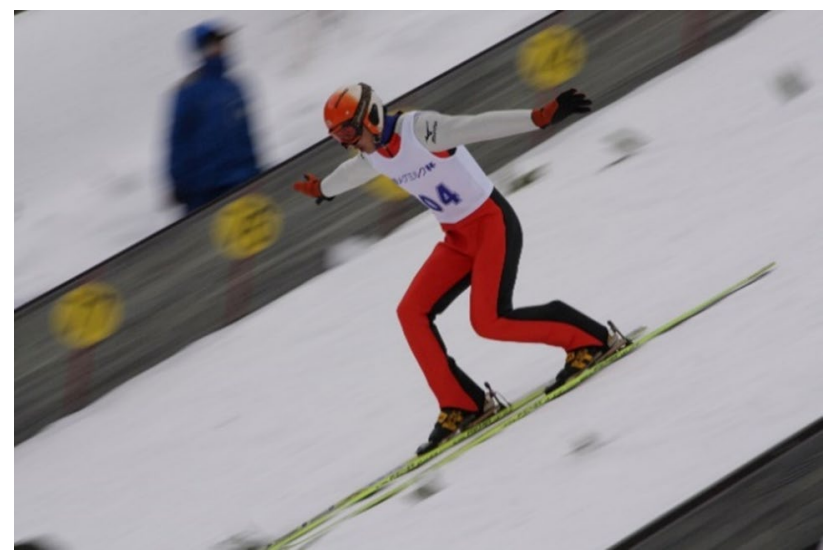

Fig. 2 Ski jumper doing a Telemark landing in Miyanomori ski jumping hill in Sapporo, Japan (Wikiwand, Ski jumping) [56]

and coaches. As a matter of facts, evidence is reported that the athletes consider the landing and its preparation as the least important phases, while the take-off is considered the most important [5]. On the other hand, previous experiences of bad landings and falls have been mentioned to negatively influence the psychology of the athlete before jumping [48], with connected consequences on the performance.

According to the questionnaire shared among athletes and published by Bessone [5], the athletes performed parallel leg landing in difficult conditions (strong wind, long distance, lack of visibility) being easier to perform than telemark. Eighty-one percent of the pool preferred to land during summer conditions, i.e. sliding in the in-run on ceramic tracks covered by water and landing on synthetic grass/mat. These materials are more stable to land on, since their surface remains regular after the athletes have been landed on. However, it has been observed how the low friction (static: 0.10; dynamic: 0.05) [25] between skis and snow could reduce the ground reaction force (GRF) acting on rear foot of the athlete, which is considered to protect the anterior cruciate ligament (ACL) [20].
Previous reviews about the research on ski jumping focused on the whole performance and on studies prior to $2006[42,44]$. In the present review, the focus is exclusively on the ski jump landing. The current paper deals with publications related to the biomechanics of landing and of its preparation, with an excursus on the methods employed for analyzing it and on the injury related to landing. Finally, suggestions for future research focused on the landing phase are proposed.

\section{Landing Biomechanics}

The knowledge in the field of ski jump landing biomechanics has been gained through in-field data collection, wind tunnel measurements and computer simulation. The in-field data collections have been almost totally conducted during (summer or winter) training in order not to affect the performance and the safety of the athletes. The outcomes of the studies investigated the kinetics, the kinematics, the muscular activity and the aerodynamics of the athletes.

\section{Kinematics}

As above mentioned, from a stable flight position, the athlete should prepare the landing acting on his/her position configuration. In order to achieve longer distances, ski jumpers try to postpone the start of their landing preparation as much as possible. This action is performed in order to exploit the aerodynamic effect (lift) for a longer time and it has been shown to be one of the performance factor together, for instance, with the take-off speed [23, 47] the body weight [35] and the motion [15]. In this regard, it has been demonstrated how the best athletes are also the ones keeping the flying position configuration for a longer time in comparison with the low ranked ski jumpers ( 0.16 vs. $0.36 \mathrm{~s}$ ) [17]. However, the longer the duration of the braking process, the softer is the landing [23].

The braking action is performed mainly acting on the ski position since it is connected with the angle of attack (i.e. the angle between the skis and the air stream). The larger the angle of attack, the shorter is the stopping distance. In particular, when an athlete widens the angle between skis and landing area and executes a backward rotation during the landing preparation, the braking action lengthens softening the landing. In fact, the wider the angle of attack, the greater is the pressure acting under the skis that permits to reduce the landing speed. In addition, the longer the time of the braking action and the longer the elastic properties of the skis are acting on the ski jumper, helping to decrease the GRF [23]. As a result, the lower the landing speed with which the athlete impacts the ground, the lower is the load on his/her musculo-skeletal system [3, 4, 17]. 
The athlete should find a good compromise when exploiting the aerodynamics to have longer jumps and to break down the speed that ranges between $80 \mathrm{~km} / \mathrm{h}$ and $85 \mathrm{~km} / \mathrm{h}$ while approaching the landing on a normal hill [50]. Postponing the landing preparation leads to an unstable landing, resulting in negative effect on performance and safety. In fact, being unable to perform a well-executed telemark landing, a lower technical score would be given by the referees. At the same time, the stability and balance might be influenced, leading to a possible increase of the injury risk.

In the last part of the stable flight phase (between 1.00 and $0.50 \mathrm{~s}$ before the impact), kinematic analysis showed that the hip and knee flexion/extension angles are comparable between the left and right sides, while the trunk is almost fully extended widening the cross-sectional area and improving the aerodynamics $[5,9,17]$. During landing preparation (around $0.50 \mathrm{~s}$ before the landing impact), the athlete progressively flexes his/her hip, knee and trunk and extends the ankle to assume the position described in the FIS ICR [12](Fig. 3). Then $0.10 \mathrm{~s}$ before the impact, the trunk, hip and knee flexion angles were steady, while the athlete prepared to absorb the GRF. After the impact, as required by the FIS ICR [12], the jumper flexed the trunk and the lower limb joints asymmetrically. The first reason is to perform the telemark landing, and the second one is to maintain the balance after the impact. The hip joint of the back leg extended more than the front one, and the back knee became more flexed $[5,17]$.
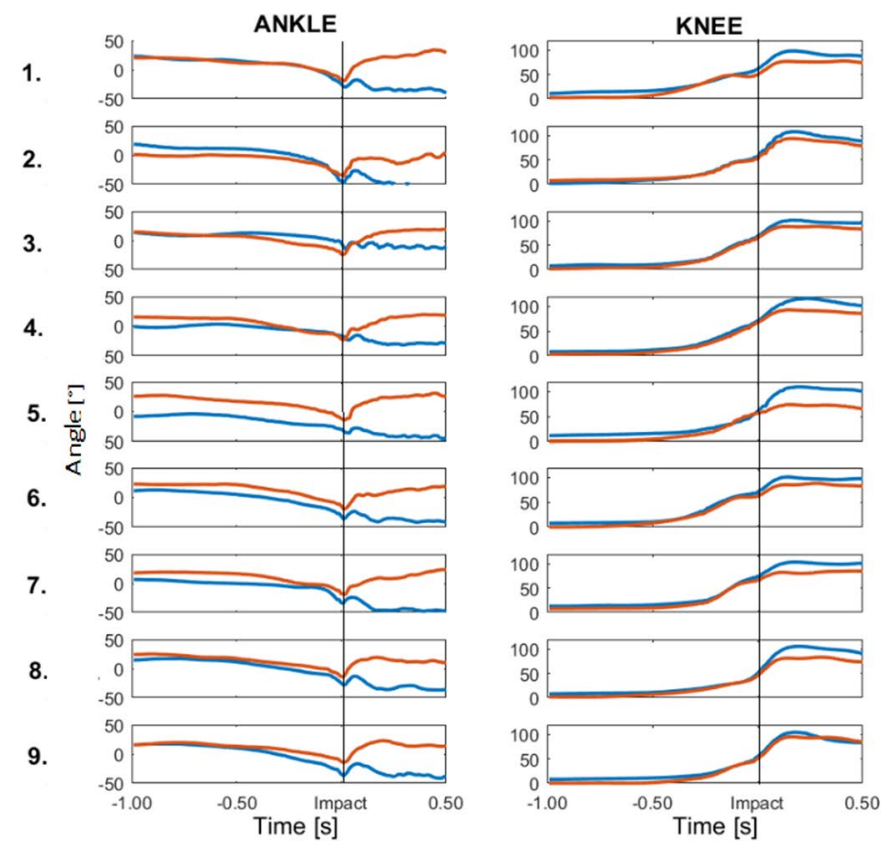

Fig. 3 Flexion/extension angles of nine jumps of one subject performing on the ski jumping hill. The angles represented are the ones of ankle, knee, hip and trunk joints. The data are reported from $1.00 \mathrm{~s}$ before the landing impact until $0.50 \mathrm{~s}$ after it. The blue line represents
Regarding the duration of the two phases, studies show how the landing preparation starts round about $0.4-0.5 \mathrm{~s}$ before the landing impact, when the main movements of the ankles and hips happen [17], followed by the ski pitch around $0.16-0.36 \mathrm{~s}$ before the impact [3]. Finally, the athlete starts moving the knees around $0.16 \mathrm{~s}$ before the impact [17].

The start (impact) and end of the landing have been defined during indoor tests by means of kinetic analysis and resemble the ones recorded during in-field tests (Fig. 4). When the force insoles detect forces higher than 0.5 body weight (BW) the landing impact happens. The end of the

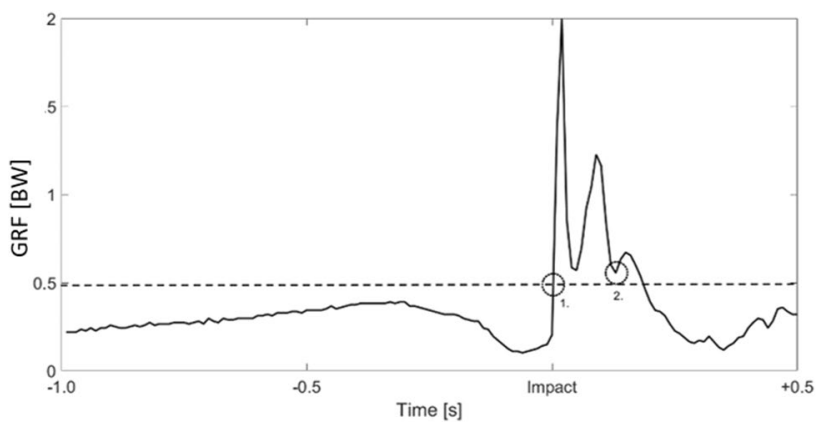

Fig. 4 An example of the normal reaction force recorded under one foot by a force insole from $1.0 \mathrm{~s}$ before the landing impact until $0.5 \mathrm{~s}$ after it. The dashed line represents 0.5 body weight, used as threshold for the start of the landing (1.), while (2.) shows the end of the landing [3]
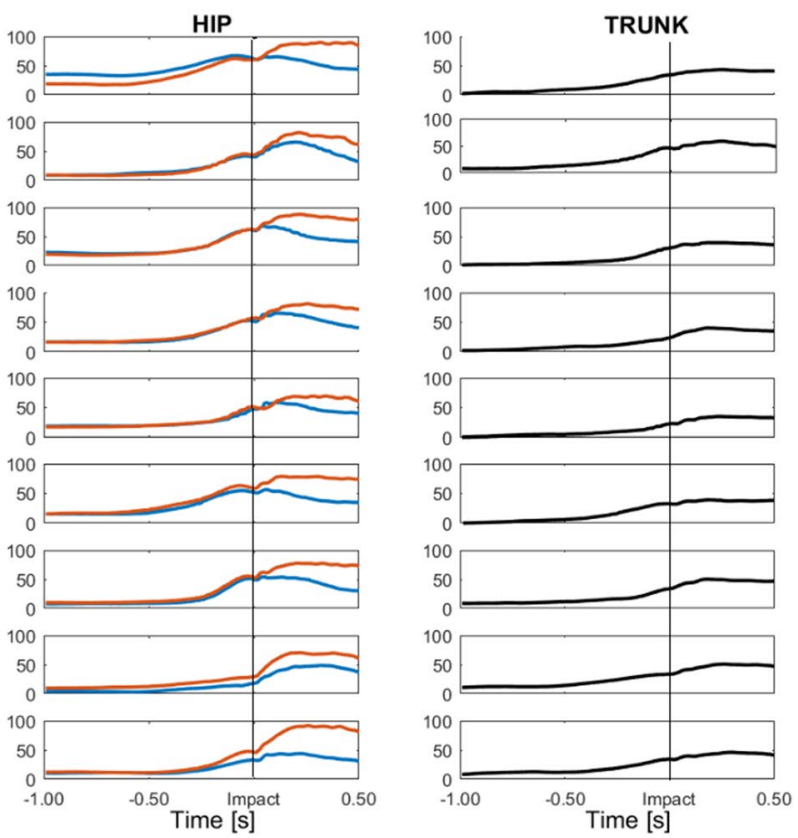

the flexion/extension of the left side joints, the red line the right ones, while the black line the one of the trunks. The black lines highlight the instant of the impact [5] 


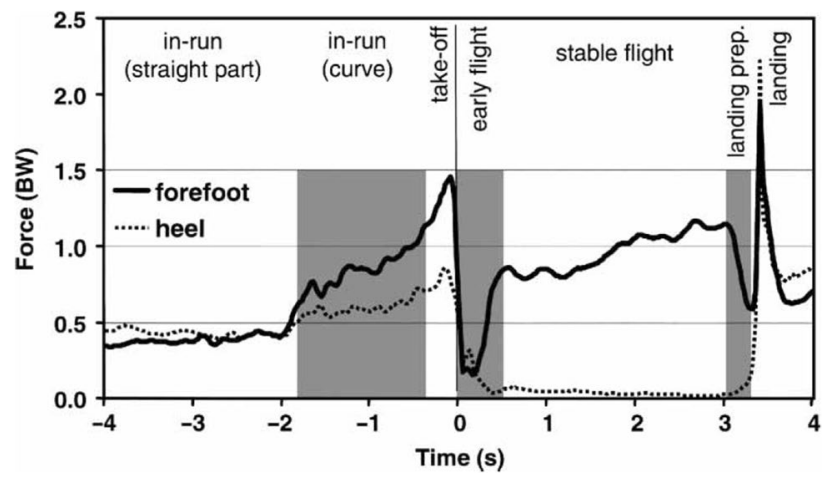

Fig. 5 Proceeding of the force from the in-run until the landing collected using pressure insoles [43]

landing coincides with the minimum of the kinetic signal following the second normal GRF peak after the impact, corresponding with the end of the eccentric phase ${ }^{1}$.

\section{Kinetics}

The first goal of evaluating the kinetics during the landing was to measure the GRF of the impact. Schwameder and Müller [41] were the first to evaluate the impact force ranging between 1.5 and 3.0 BW. Recent studies showed how the impact force per foot ranged between 1.1 and 5.3 BW [2, 4]. Not surprisingly, longer jumps correspond higher impact GRF and impulses, most probably due to the highest speed reached and due to the incline of the ski jumping hill that becomes flatter the longer the jumps are [4].

When evaluating the GRF magnitude in telemark and parallel leg landing, Bessone and colleagues [4] did not find any differences between the two executions. Therefore, it was not possible to recommend if one of the two landings guarantees lower GRF. In addition, the distribution of the force on the front and the rear foot during telemark seemed to be case specific $[3,4]$ and, in general, asymmetry between the two feet was found for $81 \%$ of the parallel landing and $50 \%$ of telemark, differently from what suggested by the FIS ICR regulations. In general, the telemark landing has been positively and negatively criticized [23], being biomechanically safer than the parallel leg landing. In fact, telemark with its step position gives more balance. On the other hand, the lack of experience of the athletes in performing the gesture could lead to an incorrect movement and therefore, to a possible injury.

Different studies showed the proceeding of the force during the entire performance [2, 41, 59] (Fig. 5). Bessone

\footnotetext{
${ }^{1}$ Fritz J, Schwameder H. [Comparison between the kinetic outcomes of the ski jumping landing impact collected by means of custom made force bindings in the field with the ones recorded indoor during imitation jumps on a force plate]. Unpublished raw data. 2017.
}

and colleagues [2] demonstrated how the trends of the analyzed ski angles (pitch and roll) are similar to the outcomes recorded by the force insoles. The result is related to the fact that the air pressure acting under the skis changes with the modification of the ski angles, and it is, consequently, detected by the force insoles.

Babiel and colleagues [1] investigated the frequency contribution of the vibrations during ski jump landing impact in comparison with alpine and cross-country skiing. The study demonstrated how the vibrations during ski jump landing have a very wide spectrum of frequency up to $15 \mathrm{~Hz}$.

As happening for normal jumps, the kinematics influences the landing kinetics. As showed in a pilot test, correlations between the hip, knee and ankle angles and the kinetic variables were found [4]. As an example, the analyzed athlete showed correlations between the GRF of the telemark front positioned leg with the front leg knee rotation, front leg hip flexion and back leg hip rotation.

\section{Electromyographic Activity}

Virmavirta and Komi [59] were the first and only scientists to perform an electromyographic analysis during the entire ski jumping performance. Focusing on the landing and its preparation, the results showed how, during the landing impact, the muscles are more active than in all the other phases, since they are involved in contrasting the GRF. In addition, muscular pre-activation was possible to notice during the landing preparation for all the investigated muscles of the lower limbs, except for the tibialis anterior (Fig. 6a). The authors discussed how an early landing preparation can be seen from the electromyographic signal that decreases in the tibialis anterior and knee extensors and that increases in the gastrocnemius and gluteus. According to Virmavirta and Komi [59], this phenomenon can be connected to the fact that the athlete is afraid to maintain the optimal flying position configuration as long as possible or that a smooth landing requires a long time to be executed. The gluteus, the tibialis anterior and the gastrocnemius showed the higher activation of the entire performance during landing (Fig. 6b). During the impact, the muscular activities of vastus medialis and lateralis, tibialis anterior, gluteus and gastrocnemius increase for damping the landing (Fig. 6a).

\section{Aerodynamics}

Ward-Smith and Clements in [52] were the first making considerations about the aerodynamics of the ski jumper approaching the landing using computer simulation and wind tunnel measurements. In particular, the focus of their study was on the ground effect, i.e. the change of aerodynamic lift acting on an object (in this case, the ski jumper) while approaching the ground [44]. 
Fig. 6 Gluteus, vastus lateralis and medialis, tibialis anterior and gastrocnemius muscle activation during the landing preparation and landing (a) and during the all performance (b) [59]

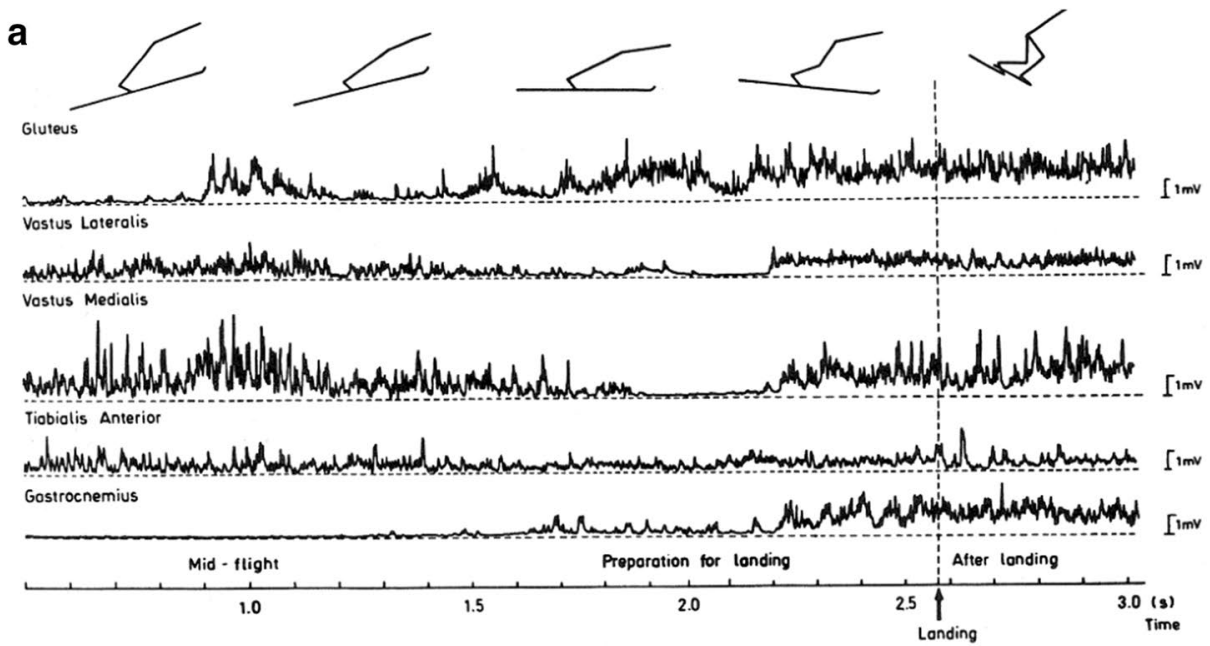

b
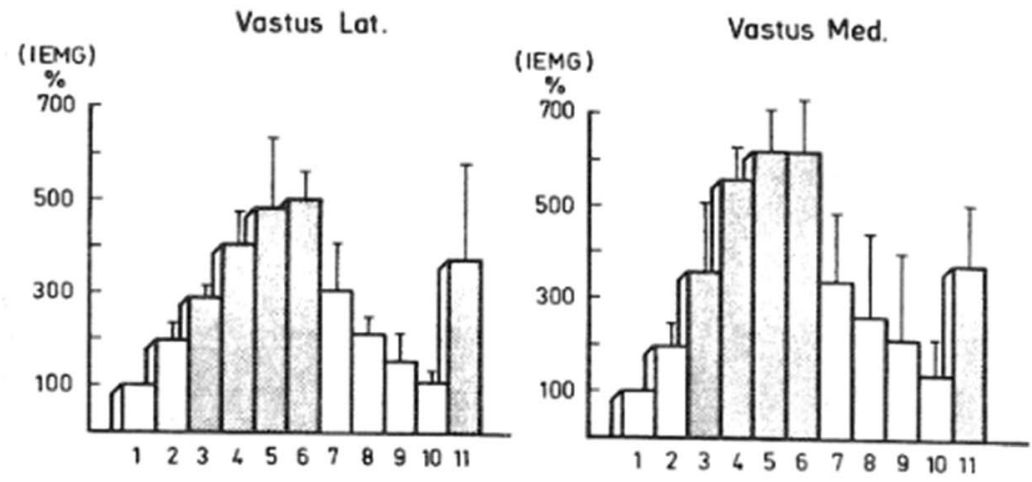

Gostrocnemius

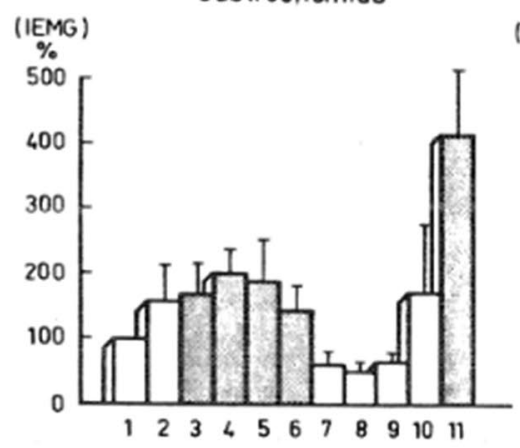

Tibiolis Ant.

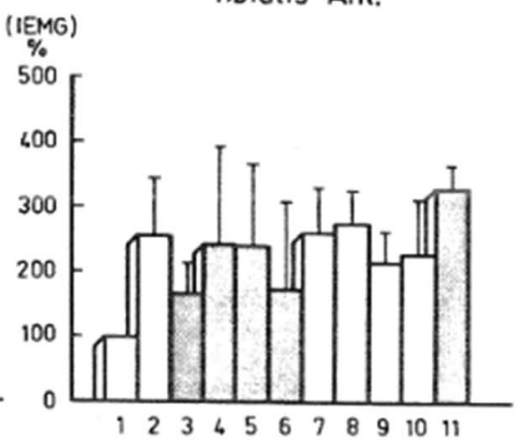

Gluteus

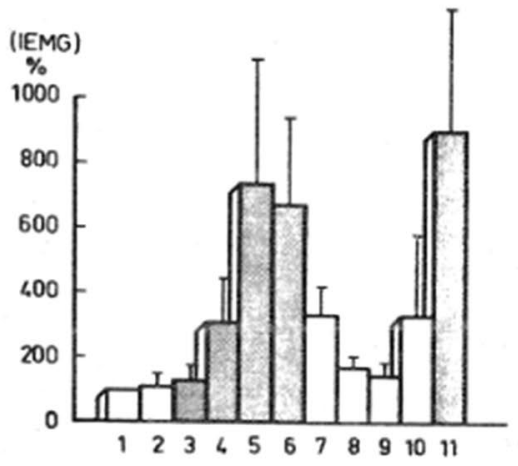


Seo and colleagues [47] demonstrated how the ground effect has a positive influence on the jump length and landing stability by means of wind tunnel tests using a dummy. Keeping the V-style for a longer time permits in fact to increase the lift action of the air, braking down the speed and improving the jump length up to three meters. In the study, the authors concentrated on the aerodynamic pitching moment, lift and drag forces acting on the athlete during the whole phase.

\section{Computer Simulation}

Computer simulations based on biomechanical model can provide valid outcomes, without risks for the athletes, and with the possibility of changing the initial conditions and the external factors acting on the system athlete plus skis. As above mentioned, Ward-Smith and Clements [52] were the first to use computer simulations to evaluate the landing biomechanics.

Müller and colleagues [41] simulated how the landing height changes when varying, for instance, the lift and drag forces, the take-off speed, the mass of the athlete or the wind acting on him/her. In the reality, changing one parameter would automatically affect the others and the athletes should optimize these changes in a short amount of time. With reference to the conditions of the study [30], the simulated landing height varied between 1.37 and $1.62 \mathrm{~m}$, while when considering data collected on the ski jumping hill, the landing height reached $2.62 \mathrm{~m}$. In general, the authors discovered that when the wind is blowing up the hill, the landing velocity is reduced, and vice versa when the wind blows from behind the athlete [33]. Finally, with the use of computer simulations, Müller and Schmölzer optimized the landing slope of the ski jumping hill considering the landing impact and height above the ground [31,32].

More recently, simulation of the trajectory using multicriterial optimization techniques forecasted how jump length and GRF are related when jumping on the normal hill of Oberstdorf, Germany $^{2}$ (Fig. 7). Firstly, it is notable how for a jump length over $95 \mathrm{~m}$ (K-point of the hill), the GRF increases drastically. In Fig. 7, the Pareto frontier (red line) for the jump length vs. the touchdown GRF was estimated from multiple optimizations with different initial conditions. For each point of the curve, an improvement of one variable can only be achieved at the cost of reducing the other one ("making it less optimal"). Using this simulation, for instance, the referees could check the current environment

\footnotetext{
${ }^{2}$ Bessone V, Petrat J, Piprek P, Fang X, Grüter B. 10.08 About optimal control methods in ski jumping (skOPTing)-Final IGSSE Project Report. Unpublished manuscript, Technical University of Munich, 2020.
}

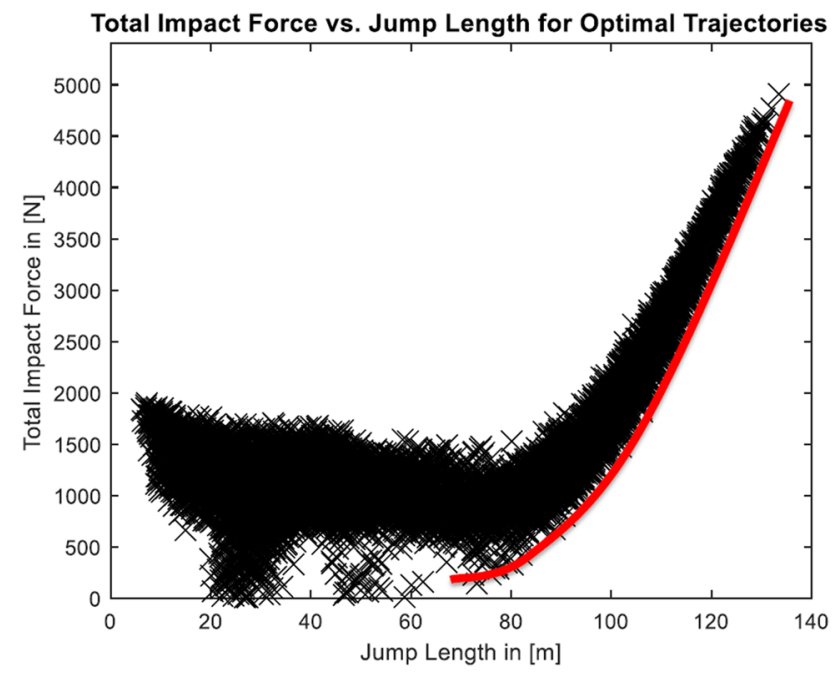

Fig. 7 Simulation of the ground reaction force acting during the impact when landing at different length by an average athlete and on the normal hill of Oberstdorf, Germany (see foonote 1). The red line stays for the Pareto frontier for the jump length vs. touchdown impact force

situation (e.g., wind) and find a suitable start gate to reduce the risk of injury during a competition.

\section{Measuring Ski Jump Landing Performance}

The ski jump landing and, in general, the overall performance is particularly challenging to biomechanically analyze during in-field data collection for different reasons, as the wide area of the hill and the small number of repetitions. Therefore, the usual in-field data collection, computer simulations of the flight phase and investigations of simulation jumps in the laboratory or in the wind tunnel are performed [42].

The main method utilized in the first studies to analyze the performance of the landing was two-dimensional video capture [17, 23]. For conducting this analysis, video cameras were placed around the landing area of the ski jumping hill. However, the high number of cameras led to a considerable time lost for their placement and calibration as well as for the video post-processing. In addition, the quality of the data was generally low since the suit of the athlete limited the detection of the joint centers [27]. On the other hand, the advantage of video capturing is that it permits to perform biomechanical analysis during competitions without interfering with the performance of the athlete [17].

To overcome the video capturing problems, in the last years, inertial motion units (IMUs) have been introduced for analyzing the kinematics of the ski jumping performance. IMUs permit, in fact, a faster set-up and a reliable threedimensional analysis $[2-6,7,9,18,26]$. Depending on the investigation, IMUs have been placed on the body of the 


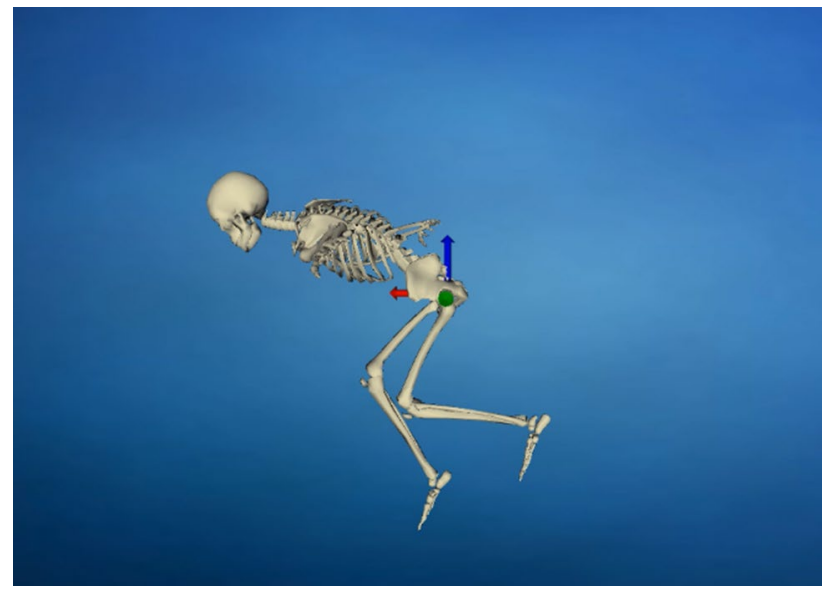

Fig. 8 Visual representation in the software iSen of the outcomes of the IMUs aktos- $t$, recorded during the landing impact (telemark position) [5]

athlete $[4,9]$ or, as an alternative, on the skis $[2,3,18]$. Thanks to the fast post-processing of the data and to a complementary dedicated software interface, a visual and objective feedback can be provided to the jumpers soon after the end of the training [5] (Fig. 8).

Chardonnens and colleagues [9] were the first ones to introduce IMUs in the ski jumping analysis, investigating the whole performance, but without specifically focusing on the landing phase. More recently, Groh and colleagues [18] introduced a new method to estimate the landing impulse and time based on inverse dynamics of data collected using IMUs. Using the acceleration recorded by IMUs and comparing the outcomes with the ones of a force binding system, i.e. custom-made ski bindings placed on a small portable force plate, the group obtained an accuracy of around $90 \%$. The method would have the advantage of analyzing the kinetics without using force measuring bindings or sensors placed in the boots, and so without interfering with the movements of the athletes.

Despite the demonstrated utility of IMUs for monitoring the ski jumping performance, some distinctions and considerations need to be made when using sensors on the whole body or on the skis and for training feedback or research. In fact if, from one side, the employment of IMUs placed on skis can be considered easy to perform during daily training, on the other side, the use of the IMUs placed on the whole body could be performed only occasionally for research or as technical feedback. The reasons are related to the positioning time, precision, calibration and cost [5]. The time necessary to position the IMUs on the whole body is longer than the placement of only two IMUs on the skis. This means that the low number of jumps performed by the athletes during a training session (from four to six), would be further reduced, being part of the training time used for placing and attaching the sensors on the athlete. Moreover, due to the precision necessary in the IMUs' placement on the whole body, a professional must perform the data collection in order to avoid positioning errors. On the contrary, the IMUs on the skis can be a cheap solution and easily placed and fixed behind the bindings by not professionals, after being correctly instructed. Finally, they can be used with a post-calibration [11], while the ones placed on the lower body need a pre-calibration before each trial, with a possible introduction of errors and time loss [5].

For the kinetic analysis of landing impact, plantar pressure insoles $[42,59,60]$ and custom-made force measuring bindings $[1,18]$ have been initially employed. However, the plantar pressure system has the disadvantage of limiting the movements due to the weight and the cables, while the custom-made bindings have the necessity of being validated after their design and are frequently heavy [43]. Thanks to the technological and connection development of the last years, new wireless force insoles have been developed and utilized in the analysis of ski jump landing, without interfering with the kinematics of the athlete $[2,3,4]$. Bessone and colleagues $[2,4]$ combined the IMUs with Bluetooth force insoles in order to detect the correlations between the kinetics and the kinematics. According to the feedback of the ski jumpers, the set a up did not influence the execution and, for this reason, it can be used for further applications during training and for biomechanical analysis.

The use of wireless force insoles is simple and even none professionals can properly use them with a high accuracy and reliability $[8,37,39,45]$. Therefore, these wearable sensors can be easily used in different locations and so an interesting solution for kinetic feedback during training camps. Furthermore, the insoles can be also employed during other kind of training, as simulation jumps. Another advantage of the wireless force insoles is that after each jump, an immediate feedback of the kinetics can be dispensed simply looking at the plot visualized on the screen of the receiver (as the iPod) [5].

Researchers can obtain a high reliability when decomposing the ski jump in easier tasks that simulate part of the performance and that are easier to analyze indoor (also in the wind tunnel) $[14,44]$. The so-called imitation jumps are simulated movements without ski equipment and in "dry" conditions and elite jumpers showed high consistency and reproducibility between the real and the imitation (take-off) [44]. Even though the validity is reduced, for instance having compromises about the speed, imitation jumps are important for training, diagnostics and research [10, 14, 28, 44]. Besides, imitation jumps increase the number of repetitions that are possible to analyze in a more reliable set-up. To the best of our knowledge, no publications dealt with imitation jumps focused on landing, even if these simulations could 
give further information about their biomechanics, as happened for the timing (see footnote 2).

The employment of wind tunnel measurements permits to efficiently estimate the aerodynamics acting on the athlete in different configurations. For this reason, the use of wind tunnel is also employed by the majority of the elite National teams for improving the flight position configuration of the athletes.

Computer simulations can predict and answer many biomechanical questions without compromising the safety of the athletes. A high number of iterations, i.e. simulated jumps, can be performed; numbers that are hard to recreate during in-field data collections. However, in order to provide reliable outcomes, the simulation needs to be based and optimized on in-field data collected. The results of the computational analysis, in fact, need to be verified and validated with real data.

\section{Injuries}

Injuries are frequent in the ski jumping World Cup circuit (around 21 every 100 jumps) [13]. However, according to the FIS Injury Surveillance System, in this sport the probability of injury is lower than in other winter disciplines [13] and severe injuries fatalities remained rare during the years $[50,51,56,55,57]$. At the same time, $65 \%$ of the overall injuries required a time-loss from training and competitions $[10,13,22,49]$. Fortunately, thanks to the technological development, the number of injuries that leads to permanent disability reduced during the time. Nowadays, this kind of injuries are rare, but during 1980s they constituted $5 \%$ of the total injuries and led to leg amputation, central nervous system lesions and blindness of the involved athletes [54].

Injuries in ski jumping are mainly occurring on the hill and during the landing phase [50]. In the publication of Stenseth and colleagues [50], 71\% of the injuries recorded during a female World Cup season happened due to a landing crash, and the adverse conditions of the in-run and out-run contributed in three out of 14 injuries. In the same study, $65 \%$ of the severe and $85 \%$ of the moderate injuries were recorded during the attempt of performing a telemark landing. Finally, $69 \%$ of the severe injuries happened in snowy or windy conditions [50]. According to the questionnaire of Bessone [5] shared among ski jumpers of different level, the athletes declared to feel unsafe in the conditions of bad grooming and lack of lightening of the landing area. These conditions are unfavorable to perform a stable landing decreasing athletes' balance [50]. Therefore, in order to decrease the number of injuries, the organizers of competitions should try to guarantee the athletes better preparation of the environmental conditions around the landing area (grooming, lightening). Finally, during the first jump of the training or competition day, but also at the beginning and at the end of the season, the probability of having injuries is reported to increase [54].

Recently, only surveys of the World Cup athletes and during the World Cup season were performed, without screening the status of the injuries of the youth and junior circuit. Especially the range between 15 and 17 years old has been showed to be more prone to injuries in the past [54]. The incidence of injury between female and male is not defined, however, regulations have been changed in favor of female athletes being more affected by ACL injuries. In particular, the hill size for female athletes has been limited at $95 \%$ of the distance of the male jumpers. According to the results of Stenseth and colleagues [50], the injuries seem to occur more frequently on shorter jumps. Therefore, the women may be safer to jump with the same regulations of their counterparts. On the other hand, the longer the jump is, the flatter is the incline of the landing area. In support of it, in the study of Bessone and colleagues [4], the recorded GRFdetected by the sensors were greater when the athlete jumped further, with possible influences on the risk of injuries.

The knee is the body part involved in most injuries (25\% of them) $[13,50]$, followed by head and face [13]. In particular, ACL rupture is a frequent knee injury in ski jumping and could be caused by the internal rotation of the knee performed during the telemark as by the high GRF during touch-down. A limitation of the internal rotation and a reduction of the GRF are, in fact, considered prevention factors in knee injuries in jumping sports $[21,29,36]$.

Besides the health problem, injuries caused staying out from competitions and training for more than four weeks in $25 \%$ of the cases. In fact, $37.5 \%$ of them involve joints and muscles, and $25 \%$ contusions [13].

Almost half of the athletes who had an injury during a competition season faced another injury the previous year [50]. Therefore, prevention programs among the teams should be considered to reduce injuries. Currently, in fact, the majority of the athletes performs rehabilitation or injury prevention exercises depending on their individual needs.

\section{Future Research}

Based on the present review, future research about ski jumping landing biomechanics is proposed. Depending on the investigation, indoor as in-field data collections can be performed. In particular, the proposed future research deals with:

Kinematic analysis should focus on the speed and the acceleration of the limbs and of the skis, during telemark and parallel leg landings. The comparison between the two 
techniques performed in equal conditions and with a comparable jump length would possibly indicate which position is the most recommendable, being biomechanically safer than the other. In addition, the center of mass position should be investigated, being an important variable in term of balance and stability. Finally, the direction of the forces and of the momenta acting on the joints can be estimated combining kinetic and kinematic data as well as inverse dynamics. The results would give additional information in regards of preventing injuries.

New equipment should be developed. The current ski jumping bindings, paddings (i.e. carbon fiber thicknesses that are placed inside the boots in order to have flatter skis during the flight phase) and boots permit a good control of the skis during the flight phase but drastically reduce the ankle range of motion during landing, with consequent unsafe movements' adaptations (as knee internal rotation) while performing the telemark. In addition, the bindings rarely release during landing with a consequent ski rotation that causes the twist of the knee. In addition, the landing is done with feet in an unnatural position that increases the strain in the knee. Finally, it has been shown how the elastic properties of the skis influence the impact kinetics [23]. Assuming that, as for alpine skiing $[34,46]$, the equipment plays an important role for injury prevention, future research should focus on its design. In particular, the focus should be on the design of boots with a greater movements' freedom, of bindings with a safe releasing when a rotation of the ski is happening during the impact and, in general, of bindings/ boots/paddings combination in order to permit a wider range of motion while performing the landing.

Wind tunnel tests could increase the understanding of the ski aerodynamics (and the system ski plus athlete in general) while approaching the landing impact. In this way, the best configuration can be found, improving the breaking action and contemporarily the jump length, acting on the angle of attack.

Landing biomechanics of female ski jumpers. This topic is missing in literature, i.e. only one focused on the flight phase [40]. However, women are generally more prone to have joints' injuries [16,24], due to anthropometrics, hormones, and neuromuscular activation. Based on any evidence, FIS is suspecting that female ski jumpers have a higher injury risk [50]. As a consequence, biomechanical analysis of the landing is recommendable to be performed on female athletes in order to better understand their biomechanics and consequently reduce the injury risk.

Computer simulations could increase the knowledge related to ski jumping and landing in particular, increasing the number of repetitions. Computer simulations could predict and answer many questions related to training methods, biomechanics, safety and health consideration without compromising the safety of the athletes, as showed by Heinrich and colleagues [20] for alpine skiing.

Electromyographic analysis could be helpful to prevent injuries. As showed by Virmavirta and Komi [58], in fact, the pre-activation of the quadriceps muscle was demonstrated to protect the ACL in other sports [19].

Imitation jumps could provide information about the biomechanics of the landing (as the telemark), while increasing the number of repetitions and reducing the risk. Besides the understanding of the biomechanics behind the telemark, imitation jumps will allow to implement exercises in order to prevent injuries.

Author Contributions VB contributed to conceptualization and methodology; VB and AS contributed to writing draft preparation and writing review and editing; VB contributed to visualization.

Funding Open Access funding enabled and organized by Projekt DEAL.

Availability of Date and Material Not applicable.

Code availability Not applicable.

\section{Compliance with Ethical Standards}

Conflict of interest The author declares to not have any conflicts of interest while publishing the article.

Open Access This article is licensed under a Creative Commons Attribution 4.0 International License, which permits use, sharing, adaptation, distribution and reproduction in any medium or format, as long as you give appropriate credit to the original author(s) and the source, provide a link to the Creative Commons licence, and indicate if changes were made. The images or other third party material in this article are included in the article's Creative Commons licence, unless indicated otherwise in a credit line to the material. If material is not included in the article's Creative Commons licence and your intended use is not permitted by statutory regulation or exceeds the permitted use, you will need to obtain permission directly from the copyright holder. To view a copy of this licence, visit http://creativecommons.org/licenses/by/4.0/.

\section{References}

1. Babiel S, Hartmann U, Spitzenpfeil P, Mester J. Ground-reaction forces in alpine skiing, cross-country skiing and ski jumping. In: Science and Skiing. Taylor \& Francis; 2003. pp. 200-7.

2. Bessone V, Petrat J, Seiberl W, Schwirtz A. Analysis of landing in ski jumping by means of inertial sensors and force insoles. Proceedings. 2008;2(6):311. https://doi.org/10.3390/proceeding s2060311.

3. Bessone V, Petrat J, Schwirtz A. Ski position during the flight and landing preparation phases in ski jumping detected with inertial sensors. Sensors. 2019;19(11):2575. https://doi.org/10.3390/ s19112575.

4. Bessone V, Petrat J, Schwirtz A. Ground reaction forces and kinematics of ski jump landing measured with wearable sensors. Sensors. 2019;19(9):2011. https://doi.org/10.3390/s19092011. 
5. Bessone V. Biomechanical analysis of landing by means of wearable sensors. Dissertation thesis. Technical University of Munich, 2020.

6. Brock $\mathrm{H}$, Ohgi Y. Assessing motion style errors in ski jumping using inertial sensor devices. IEEE Sens J. 2017;17(12):3794804. https://doi.org/10.1109/JSEN.2017.2699162.

7. Brock H, Ohgi Y. Development of an inertial motion capture system for kinematic analysis of ski jumping. Proc Inst Mech Eng Part P. 2017. https://doi.org/10.1177/1754337116677436.

8. Burns TB, Zendler JD, Zernicke RF. Wireless insoles to measure ground reaction forces step-by-step validity in hopping, walking and running. Proceedings of the 35th Conference of the International Society of Biomechanics in Sports, Cologne, GER, 14th18th June 2017.

9. Chardonnens J, Favre J, Cuendet F, Gremion G, Aminian K. A system to measure the kinematics during the entire ski jumping sequence using inertial sensors. J Biomech. 2013;46:56-62. https://doi.org/10.1016/j.jbiomech.2012.10.005.

10. Engebretsen L, Steffen K, Alonso JM. Sports injuries and illnesses during the winter olympic games 2010. Br J Sports Med. 2010;44:7727-80. https://doi.org/10.1136/bjsm.2010.076992.

11. Fang X, Göttlicher C, Holzapfel F. Attitude estimation of skis in ski jumping using low-cost inertial measurement units. Proceedings. 2018;2:317. https://doi.org/10.3390/proceedings2060317.

12. FIS. International Competition Rules (ICR) Ski Jumping. Available via: https://www.fis-ski.com/inside-fis/document-library/ ski-jumping/index.html\#deeplink=rules. Accessed 6 Sep 2017

13. Flørenes TW, Ordsletten LN, Heir S, Bahr R. Injuries among World Cup ski and snowboard athletes. Scand J Med Sci Sports. 2012;22(1):58-66. https://doi.org/10.1111/j.1600-0838.2010. 01147.x.

14. Fritz J, Kröll J, Schwameder H. Biomechanical performance diagnostics: concepts and applications in ski-jumping. 2018 36th conference of the international society of biomechanics in sports, Auckland, New Zealand, 2018.

15. Funato Y, Sakurai S. Relationship between take-off motion and jump distance in medium-hill ski jumpingTaiikugaku kenkyu. Jpn J Phys Edu Health Sport Sci. 2017;62(2):691-703.

16. Gould S, Hooper J, Strauss E. Anterior cruciate ligament injuries in females: risk factors, prevention, and outcome. Bull Hosp Jt Dis. 2013;74:146-51.

17. Greimel F, Virmavirta M, Schwameder H. Kinematic analysis of the landing phase in ski jumping. In: Müller E, Lindinger S, Stöggl T, eds. Science and Skiing IV. Aachen: Meyer \& Meyer Sport. 2009. pp. 721-7.

18. Groh BJ, Fritz J, Deininger M, Schwameder H, Eskofier BM. Unobtrusive and wearable landing momentum estimation in ski jumping with inertial-magnetic sensors. 15th international conference on wearable and implantable body sensor networks (BSN), Las Vegas, Nevada, USA. 4th-7th March 2018.

19. Hashemi J, Breighner R, Jang TH, Chandrashekar N, EkwaroOsire S, Slauterbeck JR. Increasing pre-activation of the quadriceps muscle protects the anterior cruciate ligament during the landing phase of a jump: an in vitro simulation. Knee. 1999;17(3):235-41. https://doi.org/10.1016/j.knee.2009.09.010.

20. Heinrich D, den Nachbauer BAJ, Nachbauer W. Relationship between jump landing kinematics and peak ACL force during a jump in downhill skiing: a simulation study. Scand J Med Sci Sports. 2014;24(3):180-7. https://doi.org/10.1111/sms.12120.

21. Hewett TE, Sultz SJ, Griffin LY. Understanding and Preventing Noncontact ACL Injuries. Champaign: Human Kinetics; 2007.

22. Hisdal E, Bahr R. FIS injury surveillance system 2006-2018. FIS, Fédération Internationale de Ski; Report No: 12. 2018. Available via: www.fis-ski.com. Accessed on 16 Sep 2019.

23. Hochmuth G. Telemark Landing. FIS Bull. 1999;137:29-43.
24. Ireland ML. The female ACL: Why is it more prone to injury? Orthop Clin North Am. 2002;33(4):637-51.

25. Johnson CV. Friction. Microsoft encarta online encyclopedia. 2007. Available online at https://hypertextbook.com/facts/2007/ TabraizRasul.shtml. Accessed 12 May 2020.

26. Kreibich S, Müller S, Krüger A. Sensor based measurement of ski orientation angles in ski jumping. Müller E, Lindinger S, Stöggl T, Eds. Science and Skiing IV. Aachen: Meyer \& Meyer Sport. 2012. pp. 608-18.

27. Logar G, Munih M. Estimation of joint forces and moments for the in-run and take-off in ski jumping based on measurements with wearable inertial sensors. Sensors. 2015;15(5):11258-76. https:// doi.org/10.3390/s150511258.

28. Lorenzetti S, Ammann F, Windmüller S. Conditioning exercises in ski jumping: biomechanical relationship of squat jumps, imitation jumps, and hill jumps. Sports Biomech. 2017;2:221-312. https:// doi.org/10.1080/14763141.2017.1383506.

29. Louw Q, Grimmer K. Biomechanical factors associated with the risk of knee injury when landing from a jump. S Afr J. 2006;18(1):18-23. https://doi.org/10.17159/2078-516X/2006/ v18i1a248.

30. Müller W, Platzer D, Schmölzer B. Scientific approach to ski safety. Nature. 1995;375(6531):455.

31. Müller W. Biomechanics of ski-jumping: scientific jumping hill design. In: Müller E, editor. Science and skiing. London: E\&FN Spon (Chapman \& Hall); 1997. pp. 36-48.

32. Müller W, Schmölzer B. The new jumping hill in Innsbruck: designed by means of flight path simulations. Proceedings of the IVth World Congress of Biomechanics. Calgary /AB): University of Calgary, Faculty of Kinesiology. Aug 4-9, 2002.

33. Müller W. Determinants of ski-jump performance and implications for health, safety and fairness. Sports Med. 2009;39(2):85106. https://doi.org/10.2165/00007256-200939020-00001.

34. Natri A, Beynnon BD, Ettlinger CF, Johnson RJ, Shealy JE. Alpine ski bindings and injuries Current findings. Sports Med. 1999;28(1):35-48. https://doi.org/10.2165/00007256-19992 8010-00004.

35. Oggiano L, Setran L. Effects of body weight on ski jumping performances under the new FIS rules. In: Proceedings of the 7th ISEA conference, Biarritz, FRA. 2008. https://doi.org/10.1007/ 978-2-287-09411-8_1

36. Ortega DR, Bies EC, Berral de la Rosa FJ. Analysis of the vertical ground reaction forces and temporal factors in the landing phase of a countermovement jump. J Sports Sci \& Med. 2010;92:822-87.

37. Peeble AT, Maguire LA, Renner RK, Queen RM. Validity and repeatability of single-sensor loadsol insoles during landing. Sensors. 2018;18(12):40-82. https://doi.org/10.3390/s18124082.

38. Ranta M, von Hetzenvon R. Landing in ski jumping: An expert report for the FIS Technical Board. Oberhofen am Thunersee: FIS. 1999.

39. Renner RK, Williams BDS, Queen RM. The reliability and validity of the loadsol ${ }^{\circledR}$ under various walking and running conditions. Sensors. 2019;19:22-65. https://doi.org/10.3390/s19020265.

40. Sasaki H, Tsunoda K, Hoshino H, Maeda K, Koike T, Sasaki T. Physical characteristics of Japanese female and ski jumpers In: Müller E, Bacharach D, Klika R, Lindinger S, Schwameder H, eds. Science and Skiing III. Meyer \& Meyer Sport. 2009.

41. Schwameder H, Müller E. Biomechanische Beschreibung und Analyse der V-Technik im Skispringen (Biomechanical description and analysis of the $\mathrm{V}$-technique in ski-jumping). Spectrum der Sportwissenschaften 1995;7:5-36.

42. Schwameder H, Müller E. Biomechanics in ski-jumping: A review. Eur J Sport Sci. 2001;1:1. https://doi.org/10.1080/17461 390100071107. 
43. Schwameder H. Biomechanics research in ski jumping, 19912006. Sports Biomech. 2008;71(1):114-136. https://doi.org/10. 1080/14763140701687560

44. Schwameder H. Concepts in ski jumping biomechanics and potential transfer to other sports. Proceedings of the 32nd International Congress of Biomechanics in Sports, 12th-16th July 2014.

45. Seiberl W, Jensen E, Merker J, Leitel M, Schwirtz A. Accuracy and precision of loadsol ${ }^{\circledR}$ insole force-sensors for the quantification of ground reaction force-based biomechanical running parameters. Eur J Sport Sci. 2018;18(8):1100-9. https://doi.org/10.1080/ 17461391.2018.1477993.

46. Senner V, Michel FI, Lehner S. Ski equipment-related measures to reduce knee injuries. Available via: https://www.bfu.ch/sites/ assets/Shop/bfu_2.119.08_bfu\%20knowledge\%20base\%20\%E2\% $80 \% 93 \% 20$ Ski $\% 20$ equipment-related $\% 20$ measures $\% 20$ to $\% 20$ red uce\%20knee\%20injuries.pdf. Accessed 10 July 2019.

47. Seo K, Watanabe T, Igarashi M, Kimura S, Murakami M. Aerodynamic study for the ground effect of ski jumping. In: Proceedings of the 19th international symposia on biomechanics in sports, San Francisco, USA. 2001.

48. Sklett VH, Lorås HW, Sigmundsson H. Self-efficacy, flow, affect, worry and performance in elite World Cup ski jumping. Frontiers in Psychology. 2018. https://doi.org/10.3389/fpsyg.2018.01215.

49. Soligard T, Steffen K, Palmer-Green D, Aubry M, Grant ME, Meeuwisse W, Mountjoy M, Budgett R, Engebretsen L. Sports injuries and illnesses in the sochi 2014 Olympic winter games. Br J Sports Med. 2015;49:441-711. https://doi.org/10.1136/bjspo rts-2014-094538.

50. Stenseth OMR, Barli SF, Martin RK, Engebretsen L. Injuries in elite women's ski jumping: surveillance through the 2017-18 FIS
World Cup season. Br J Sports Med. 2020;54(1):44-48.https://doi. org/10.1136/bjsports-2019-100799.

51. Stillhard A, Buschor C, Krastl G, Kühl S, Filippi A. Frequency of injuries, in particular dental injuries, in Ski jumping and Nordic combined. Swiss Dent J. 2015;12(5):815-9.

52. Ward-Smith A, Clements D. Experimental determination of the aerodynamic characteristics of ski jumpers. Aeronaut $\mathrm{J}$. 1982;8(6):384-91.

53. Watanabe T, Kasaya A, Kawahara,Y. Kinematic studies on ski jumping: Proceedings of the International Congress of Winter Sports Medicine. Sapporo: JPN. 1972. pp. 98-105

54. Wester K. Serious Ski jumping injuries in Norway. Am J Sports Med. 1985;13:124-7.6.

55. Wester K. Improved safety in Ski jumping. Am J Sports Med. 1988;16:499-500.

56. Wikiwand and, Ski jumping. Available online: https://www.wikiw and.com/en/Ski_jumping. Accessed on March 9, 2020

57. Wright JR. Nordic ski jumping fatalities in the United States [J]. J Trauma. 1988;28(6):848-51.

58. Virmavirta M, Komi PV. Electromyographic analysis of muscle activation during ski jumping performance. Int J Sport Biomech. 1991;7:175-82.

59. Virmavirta M, Komi PV. Plantar pressures during ski jumping take-off. J Appl Biomech. 2000;16:320-6. https://doi.org/10.1123/ jab.16.3.320.

60. Virmavirta M, Perttunen J, Komi PV. EMG activities and plantar pressures during ski jumping take-off on three different sized hills. J Electromyogr Kinesiol. 2001;11(2):141-7. 